\title{
Molecular docking to explore the possible binding mode of potential inhibitors of thioredoxin glutathione reductase
}

\author{
JINGWEI HUANG $^{1 *}$, WEIJUAN HUA ${ }^{2 *}$, JIAHUANG LI $^{1}$ and ZICHUN HUA ${ }^{1,3}$ \\ ${ }^{1}$ The State Key Laboratory of Pharmaceutical Biotechnology, College of Life Sciences, Nanjing University, \\ Nanjing, Jiangsu 210093; ${ }^{2}$ Department of Biology, Jiangsu Second Normal University, Nanjing, Jiangsu 210013; \\ ${ }^{3}$ Changzhou High-Tech Research Institute of Nanjing University and Jiangsu Target Pharma Laboratories Inc., \\ Changzhou, Jiangsu 213164, P.R. China
}

Received September 4, 2014; Accepted June 22, 2015

DOI: $10.3892 / \mathrm{mmr} .2015 .4119$

\begin{abstract}
Praziquantel (PZQ) is the treatment of choice for schistosomiasis, one of the most important but neglected tropical diseases. Recently, however, Schistosoma have exhibited reduced susceptibility to PZQ, and an urgent need to develop new drugs to treat schistosomiasis has emerged. Thioredoxin glutathione reductase (TGR) plays a crucial role in the redox balance of the parasite, combining glutaredoxin (Grx), glutathione reductase and thioredoxin reductase (TR) activities. Several compounds, including oxadiazole 2-oxides, phosphinic acid amides, isoxazolones and phosphoramidites, have been identified as agents that inhibit TGR from Schistosoma mansoni (smTGR) and exhibit anti-schistosomal activity. 4-Phenyl-1,2,5-oxadiazole-3-carbonitrile-2-oxide has also been shown to be active against TGR from Schistosoma japonicum (sjTGR). The binding sites of these inhibitors, however, remain unclear. To explore the binding interactions of these compounds, we selected six compounds to dock into the NADPH binding site, the active site of the TR domain and the Grx active site of both smTGR and sjTGR using AutoDock 4.2.5.1. The results suggested that the most favoured binding site for all compounds in either sjTGR or smTGR was the oxidised glutathione-binding pocket of the TR domain. Although all of the compounds could fit into the sjTGR site, the inhibition efficiency of these compounds towards sjTGR was marginally lower than it was towards smTGR,
\end{abstract}

Correspondence to: Professor Zichun Hua or Dr Jiahuang Li, The State Key Laboratory of Pharmaceutical Biotechnology, College of Life Sciences, Nanjing University, 22 Han Kou Road, Nanjing, Jiangsu 210093, P.R. China

E-mail: huazc@nju.edu.cn

E-mail: 1ijiah@nju.edu.cn

*Contributed equally

Key words: molecular docking, thioredoxin glutathione reductase from Schistosoma japonicum, thioredoxin glutathione reductase from Schistosoma mansoni, potential inhibitors, binding mode suggesting that it would be necessary to design specific inhibitors of TGR for different Schistosoma species. The docking results showed that all compounds docking in SmTGR and sjTGR adopted similar binding modes in the TR domain. Two peptide fragments from another subunit, Phe505'-Leu508' and Pro572'-Thr577', played a critical role in the interactions with the inhibitors. In conclusion, the present study has revealed binding mechanisms for potential inhibitors of Schistosoma TGRs and could lead to structure-based ligand design and the development of new anti-schistosomiasis drugs.

\section{Introduction}

Schistosomiasis is a major infectious tropical disease caused by blood flukes (trematode worms) of the genus Schistosoma. Following malaria, schistosomiasis is the second most socioeconomically devastating parasitic disease in the world. The disease is prevalent in East and Southeast Asia, Africa and Latin America and affects > 200 million individuals each year (1). There are three major species of Schistosoma worms that parasitize the human body: mansoni, japonicum and haematobium (2).

In East and Southeast Asia, including China, where schistosomiasis is a serious problem, the prevalent species of the parasite is Schistosoma japonicum (3). The first drug that was shown to be effective against schistosomiasis, in 1918, was antimony potassium tartrate. Praziquantel (PZQ) was discovered in the mid-1970s and has effectively been the only drug used for the large-scale treatment of schistosomiasis since its discovery (4). Due to this, however, parasites with low susceptibility to PZQ have begun to emerge $(5,6)$, thus making the development of new drugs for the treatment of schistosomiasis an urgent necessity.

Thioredoxin glutathione reductase (TGR) plays a crucial role in maintaining redox homeostasis in the parasite (7). TGR is a homodimeric flavoprotein in which each subunit comprises a glutaredoxin (Grx) domain fused to a typical thioredoxin reductase (TR) domain. The TR domain is analogous to the glutathione reductase (GR) domain: Both the TR and GF enzymes belong to the same superfamily of dimeric flavoenzymes, and share similar global folds, cofactors (FAD), substrate binding sites and active site residues, and have 
similar catalytic mechanisms. Schistosoma has lost the genes encoding TR and GR (two of the main detoxification pathways in mammals) and depends on the single TGR enzyme, which combines the enzymatic activities of GR, TR and Grx, to control redox homeostasis. Adult parasites are killed by RNA interference gene silencing of TGR, confirming TGR as a potential drug target for the treatment of schistosomiasis (8).

The overall structure of TGR from Schistosoma mansoni (smTGR) is a fusion of two domains: Grx (residues 1-106) and TR (residues 107-598) (9). The active cavity of the TR domain comprises residues from both subunits: An FAD-binding motif and a redox-active Cys154-Cys159 pair from one subunit, and a C-terminal domain containing a conserved redox-active four peptide fragment tail (-Gly595'-Cys596'-Sec597'-Gly598') from the adjacent subunit. The NADPH binding site is located in the middle of the TR domain, close to the FAD-binding site and the thiol/disulphide redox active centre Cys154-Cys159.

The proposed electron flow within the TGR protein is from NADPH to the thiol/disulphide Cys154-Cys159 pair that forms the redox-active centre, and then to the C-terminus and, finally, from the C-terminus to the Grx active site (Cys28-Cys31) or thioredoxin $(10,11)$. Three binding site cavities within the TGRs therefore appear to be important for electron delivery: i) The GSH binding site in Grx; ii) the NADPH binding site and iii) the TR active cavity, which contains the FAD-binding site and a redox-active Cys154-Cys159 pair from one subunit, and a redox-active C-terminus from the other subunit. Inhibitors occupying these sites may disrupt electron delivery within the TGR proteins.

It has been reported in studies by Doenhoff et al (4), Simeonov et al (12) and Sayed et al (13) that several compound groups, including oxadiazole 2-oxides, phosphinic acid amides, isoxazolones and phosphoramidites, inhibit smTGR. 4-Phenyl-1,2,5-oxadiazole-3-carbonitrile-2-oxide (termed compound 1 in the present study) has been found to inhibit both the TR and GR activities of smTGR in the low nanomolar range and has also been shown to be active against TGR from Schistosoma japonicum (sjTGR) (13). The binding sites of these prototype inhibitors of TGR, however, remain unclear.

To explore how these inhibitors interact with the TGRs, we docked six compounds from the four groups described above into the Grx domain, the NADPH-binding site and the TR active site cavity of sjTGR and smTGR using AutoDock 4.2.5.1. (The Scripps Research Institute, La Jolla, CA, USA) (14). Knowledge of the possible binding sites is likely to enhance the understanding of the inhibitory mechanisms and facilitate the development of new anti-schistosomiasis drugs.

\section{Materials and methods}

Homology modelling of sjTGR. The three-dimensional (3D) structure of sjTGR has not yet been elucidated; however, since the sequence homology between sjTGR and smTGR is $89 \%$, the sjTGR structure can be modeled on that of smTGR, for which an X-ray structure has been determined.

The amino acid sequence of sjTGR was extracted from UniProtKB/TrEMBL (B5THG7), and the smTGR $\mathrm{X}$-ray structure [Protein Data Bank (PDB) ID: 2X99, resolutions $2.3 \AA$ ] (9) was used as a template. Homology modelling of the sjTGR subunit was performed using
Discovery Studio 2.5 (Accelrys Software, Inc., San Diego, CA, USA). The sjTGR dimer was assembled using PISA (http://www.ebi.ac.uk/msd-srv/prot_int/pistart.html) (15).

Protein structure analysis and graphical display were performed using Discovery Studio View 4.0 (Accelrys Software, Inc.). The derived protein structure was then evaluated using the web-based software PROCHECK (http://www. ebi.ac.uk/pdbsum/) $(16,17)$.

Chemistry. The six inhibitor molecules selected for this study could be divided into four groups: The oxadiazole 2-oxides (compounds 1-3), a phosphinic acid amide (compound 4), an isoxazolone (compound 5) and a phosphoramidite (compound 6) (Table I). Two-dimensional structures of all drug molecules were sketched using ISIS Draw 2.5 (MDL Information Systems, Inc., San Leandro, CA, USA) and converted into optimised 3D structures using Discovery Studio 2.5 (Accelrys Software, Inc.). All six compounds have been reported to inhibit smTGR (4,12,13), and 4-phenyl-1,2,5-oxadiazole-3-carbonitrile-2-oxide (compound 1) has also been reported to inhibit sjTGR in vitro $(4,12)$.

The native substrates, glutathione (GSH), oxidised glutathione (GDS) and NADPH, were also docked into the corresponding sites as controls. GDS, the specific substrate of GR, was extracted from human GR (PDB ID: 2GRT) (18). GSH and NADPH were extracted from smTGR (PDB ID: 2X99) (9).

Molecular docking. Molecular docking was performed using AutoDockTools 4.2.5.1 (The Scripps Research Institute) (14). The AutoDockTools graphical interface AutoDockTools 1.5.6 was used to preserve polar hydrogen atoms and to add partial charges to the proteins using Gasteiger charges (14). AutoGrid was used to generate the grid maps. Each grid was centred at the binding site of the protein. The grid dimensions were 40 points in each dimension separated by $0.375 \AA$ (except NADPH for 40x40x60). For all ligands, random starting positions, orientations and torsions were used. Default values in AutoDock were used for the translation, quaternion and torsion steps. The Genetic Algorithm was used with 2,500,000 energy evaluations and a population of 300 individuals; 100 runs were carried out. Following docking, the results were clustered into groups with root-mean-square deviation $<2.0 \AA$. The ranking of the clusters was performed based on the lowest energy representative of each cluster. Interactive visualisation and analysis of molecular structures and interactions between protein and ligand were performed using Discovery Studio View 4.0 software (Accelrys Software, Inc.)

\section{Results and Discussion}

Homology modelling and structure evaluation. There is $89 \%$ identity between the template smTGR (smTGR 2x99) and the sjTGR sequences. Due to the fact that the template sequence lacks the $\mathrm{N}$ - and $\mathrm{C}$-terminal $\mathrm{SeC}$ tail peptide fragments, the model of sjTGR that was used contained only residues 6-592 (Fig. 1A). The coincidence degree between the sjTGR model and the template is shown in Fig. 1B. The shades of the colours, from dark green to grey, represent the model's degree of coincidence, from low to high. The unmatched region was mainly located at the C-terminal; other regions of the model showed 
Table I. Selected inhibitors for docking.

\begin{tabular}{|c|c|c|c|c|}
\hline Compound index & Core structure formula & R1 & $\mathrm{R} 2$ & Ref. \\
\hline 1 & & $-\mathrm{CN}$ & $-\mathrm{Ph}$ & $(11,12)$ \\
\hline 2 & & & & $(11,12)$ \\
\hline 3 & & NA & NA & $(11,12)$ \\
\hline 4 & & & NA & $(11,12)$ \\
\hline 5 & & & NA & (11) \\
\hline 6 & & Naphthyl & NA & (11) \\
\hline
\end{tabular}

Compounds 1-3 are oxadiazole 2-oxide analogues. Compound 4 is a phosphinic acid amide analogue. Compound 5 is an isoxazolone analogue. Compound 6 is a phosphoramidite analogue. All compounds were identified as effective inhibitors of thioredoxin glutathione reductase from Schistosoma mansoni. NA, not applicable.

high coincidence with the template (Fig. 1B). The rationality of the stereochemistry in the newly constructed model was evaluated using PROCHECK. The $\phi-\psi$ plot of the sjTGR dimer is shown in Fig. 1C. With the exception of the Gly and Pro residues, there were 916 amino acids (89.5\%) in the most favoured regions and 104 amino acids (10.2\%) in additional allowed regions. G-factor values of all dihedral angles were $>-0.5$, which suggested that the overall structure was reasonably good (Fig. 1C).

Molecular docking. Six compounds, belonging to four different groups, were docked separately into the Grx and TR active sites and the NADPH binding site. The free energy of binding, comprising van der Waals, electrostatic and hydrophobic interactions and solvation energy, was used to evaluate the binding affinity. The docked conformations were clustered by energy and the conformation with the lowest free binding energy was selected for further analysis. As controls, GSH (a native substrate of the Grx domain), GDS (a native substrate that can bind in the GR/TR active site) and NADPH were also docked/redocked into the appropriate sites for comparison.

Native substrates in the corresponding sites. The structure of a TGR in complex with a substrate/inhibitor in the thiol/disulphide redox active pocket (TR active cavity) has not yet been determined. The TR domain of TGR has similarities with TRs and GRs and exhibits TR and GR enzymatic activities. Since the GDS binding pocket close to the FAD-binding site (thiol/disulphide redox active centre) has been proposed to play a major role in the reduction of GDS in GR, TR and smTGR $(19,20)$, GDS was docked into the TR active cavity of smTGR and sjTGR, based on the structure of the human GR-GDS complex (PDB ID: 2GRT) (18). It was found that GDS was embedded into the dimer interface, near the FAD-binding site and the disulphide motif (Cys154-Cys159). The binding site for GDS in the TR active cavity of TGR was observed to be composed of residues from two subunits. In smTGR, GDS was surrounded by Ser117, Ala121, Lys124, Val155, Ile160, Lys163, His204, Leu208, Tyr212, Ile446 and Arg450 from one subunit and Lys506', Leu508', His571', Pro572', Thr573', Cys574', Glu576', Thr577' and Thr580' from another subunit. As observed in docking results reported by Sharma et al (20), GDS in the active site formed hydrogen bonds with Tyr212, Lys124 and Arg450, residues that are also conserved in GR. GDS also formed hydrogen bonds with Leu508', His571', Thr573' and Glu576' from the other subunit.

In sjTGR, GDS occupies the same position as in smTGR, except that Leu120 replaces the Lys124 that interacts with GDS in smTGR (Fig. 2A). The remaining residues that form contacts with GDS in sjTGR are the same as those described 

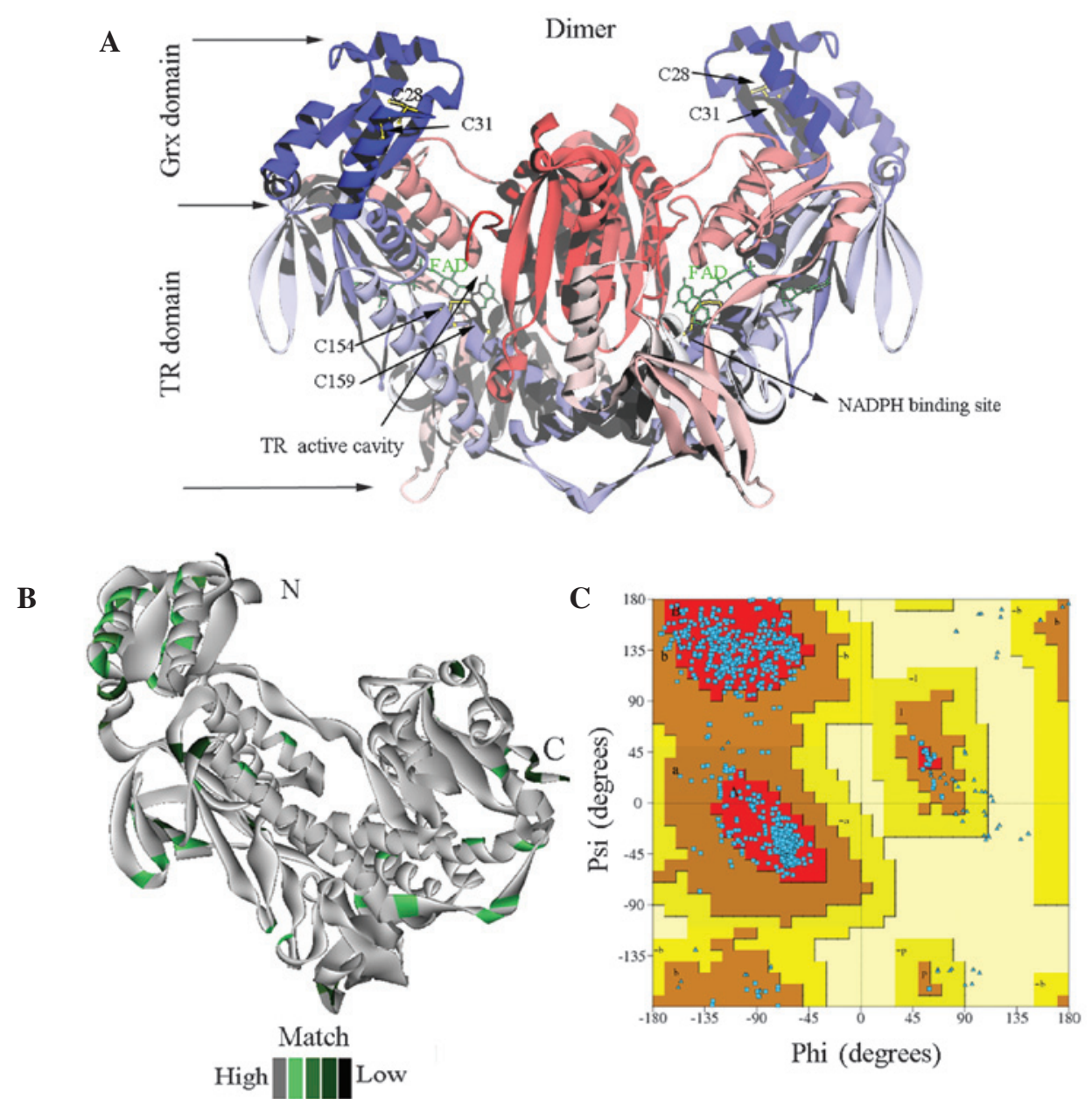

Figure 1. (A) Homology structure of sjTGR dimer. (B) Comparison between the sjTGR homology model and the template of thioredoxin glutathione reductase from Schistosoma mansoni. Colours stand for each model's degree of coincidence, from low to high (from dark to light, respectively). (C) Ramachandran plot of the monomeric sjTGR model and results of protein structure evaluation of the sjTGR model using PROCHECK. TR, thioredoxin reductase; Grx, glutaredoxin; sjTGR, thioredoxin glutathione reductase from Schistosoma japonicum.

for smTGR. The estimated free energy of binding of GDS to smTGR is $-2.80 \mathrm{kcal} / \mathrm{mol}$, compared with $-3.39 \mathrm{kcal} / \mathrm{mol}$ for binding to sjTGR (Tables II and III), indicating that GDS binding to sjTGR is more stable than GDS binding to smTGR.

One reduced glutathione (GSH) was found to bind in the active site of the Grx domain of the smTGR template (PDB ID 2x99) (9), surrounded by three segments of the polypeptide, residues Lys25-Tyr30, Thr71-Pro73 and Asp84-Glu86. It was also found that Gln60 could form a hydrogen bond with GSH. As a control, GSH was re-docked into the Grx domain of smTGR and it was found that the docked molecule completely overlapped with the GSH conformation observed in the X-ray structure. As with smTGR, GSH in sjTGR also made contacts with the conserved residues Lys25, Cys28, Gln60, Thr71, Val72, Pro73, Asp84 and Ser85 (Fig. 2B). In sjTGR, residues Phe30 and Lys86 replace Tyr30 and Gln86 of smTGR, respectively. These replacements resulted in an increase in free binding energy from $-4.14 \mathrm{kcal} / \mathrm{mol}$ in smTGR to $-3.17 \mathrm{kcal} / \mathrm{mol}$ in sjTGR (Tables II and III), indicating that the Grx domains of the two TGRs have different binding capacities for GSH.

NADPH functions as an electron donor in the electron transport chain of TGRs. An X-ray structure of smTGR in complex with NADPH (9) showed the NADPH was surrounded by residues Lys162, Pro264, Ile292-Ala298, Glu300, Met315, Arg317-Ile319, Leu321, Arg322, Pro349, Glu390-Arg393, Gln440, Leu441, Thr472 and Phe474. The present docking results showed that NADPH could re-dock into its initial position with an estimated free binding energy of $-10.17 \mathrm{kcal} / \mathrm{mol}$. In sjTGR, the docked NADPH interacts with the conserved residues Lys162, Pro264, Gly293-Ala298, Lys300, Arg317-Ile319, Leu321, Arg322, Ala390-Arg393, Gln440, Leu441 and Thr472-Phe474 (Fig. 2C). Although the NADPH binding site appears to be conserved among TGRs, the estimated free binding energy with sjTGR $(-6.59 \mathrm{kcal} / \mathrm{mol})$ was significantly higher than that with smTGR.

The present data suggested that sjTGR and smTGR have different binding capacities for their native substrates, despite the fact that they share high sequence identity and conserved residues in their binding sites. Our previous kinetic analysis (21) showed that the Km value for sjTGR with GDS $(49.55 \mu \mathrm{M})$ was lower than that for smTGR $(71.5 \mu \mathrm{M})$, whereas the Km values for sjTGR with NADPH $(21.426 \mu \mathrm{M})$ and GSH $(1,698 \mu \mathrm{M})$ were higher than those for smTGR (13.7 and $248.6 \mu \mathrm{M}$, respectively). This indicates that sjTGR has a higher binding capacity for GDS than does smTGR, while the binding capacities of sjTGR for GSH and NADPH were lower 
Table II. Estimated free energy of binding of drugs and native substrates with thioredoxin glutathione from Schistosoma mansoni.

Estimated free binding energy $(\mathrm{kcal} / \mathrm{mol})$

\begin{tabular}{lccc}
\cline { 2 - 3 } Ligand & Grx active site & TR active cavity & NADPH-binding site \\
\hline Compound 1 & -6.19 & -6.63 & -7.15 \\
Compound 2 & -5.42 & -7.75 & -7.86 \\
Compound 3 & -4.72 & -7.03 & -7.21 \\
Compound 4 & -5.80 & -7.23 & -7.34 \\
Compound 5 & -6.14 & -7.56 & -8.46 \\
Compound 6 & -6.68 & -7.66 & -7.02 \\
GDS & & -2.80 & -10.17 \\
GSH & -4.14 & & \\
NADPH & & & \\
\hline
\end{tabular}

Grx, glutaredoxin; TR, thioredoxin reductase; GSH, glutathione; GDS, oxidised glutathione.

Table III. Estimated free binding energy of drugs and native substrates with thioredoxin glutathione from Schistosoma japonicum.

Estimated free binding energy $(\mathrm{kcal} / \mathrm{mol})$

\begin{tabular}{lccc} 
& Grx active site & TR active cavity & NADPH-binding site \\
\cline { 2 - 4 } Compound 1 & -4.73 & -6.45 & -6.50 \\
Compound 2 & -3.98 & -7.04 & -7.67 \\
Compound 3 & -4.23 & -6.86 & -7.10 \\
Compound 4 & -4.57 & -6.92 & -6.81 \\
Compound 5 & -4.63 & -7.72 & -6.48 \\
Compound 6 & -4.55 & -7.44 & -6.35 \\
GDS & & -3.39 & -6.59 \\
GSH & -3.17 & &
\end{tabular}

Grx, glutaredoxin; TR, thioredoxin reductase; GSH, glutathione; GDS, oxidised glutathione.

than those of smTGR, in agreement with our conclusion. These results are concordant with those of the present study, which suggest that sjTGR and smTGR have different binding capacities for their native substrates, despite sharing high sequence identity and conserved residues in their binding sites.

TR active site cavity as the effective binding site for inhibitors. Although the six selected compounds inhibit smTGR in vitro $(4,12,13,22)$, the mechanism by which that happens remains unknown. The present study has focused on whether these molecules can directly gain access to the three main functional sites of TGR. The binding scores of the best-docked conformations of ligands in smTGR and sjTGR are listed in Tables II and III, respectively.

In smTGR, the NADPH-binding site and TR active cavity (GDS binding site) exhibit lower estimated free binding energies for the compounds than does the Grx domain (Table II). The free binding energy shown by one of the controls, NADPH, was much lower than the free binding energy shown by all the test compounds.
In sjTGR, the NADPH-binding site and the TR active cavity exhibited better binding capacities for the test compounds than the Grx domain (Table III). In each site, the binding capacity of sjTGR for the test compounds was generally marginally weaker than that of smTGR, apart from compound 5, which exhibited a better free binding energy for the TR active cavity of sjTGR. The most marked differences in binding capacities were observed in the Grx active site, with an increase of $1.21 \mathrm{kcal} / \mathrm{mol}$ in average binding energy for each test compound.

It is not sufficient, however, to estimate the free binding energy of single test molecules alone. In order to clarify whether compounds can competitively inhibit the enzymatic activity of TGR, the free binding energy between test molecules and native substrates was compared. The ratios of the free binding energies of inhibitors to those of native substrates (docking ratios) were used to evaluate the inhibitory efficiency of the compounds.

The docking ratios of the test molecules in smTGR are shown in Fig. 3A. All docking ratio values in the TR active 
A

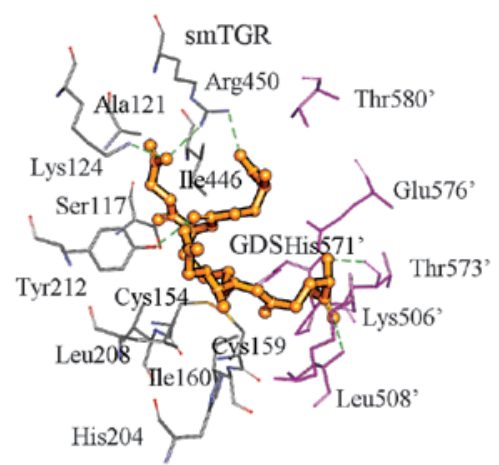

B

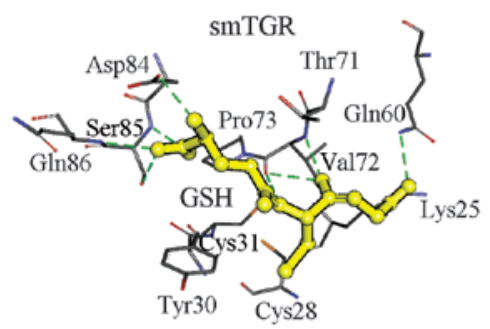

C

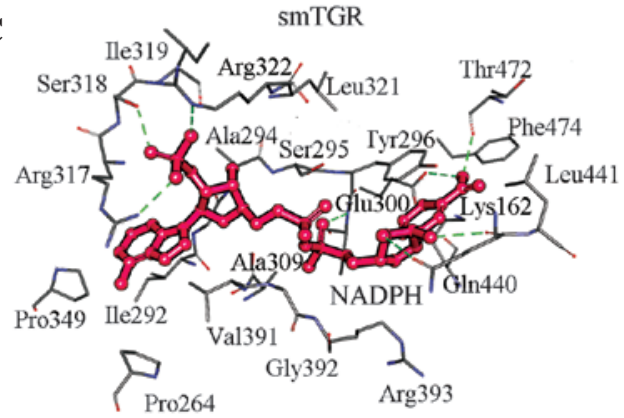

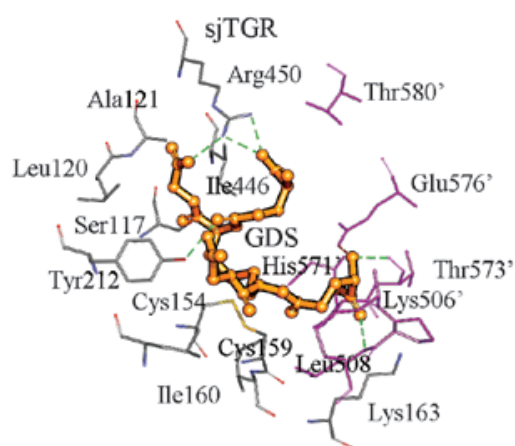
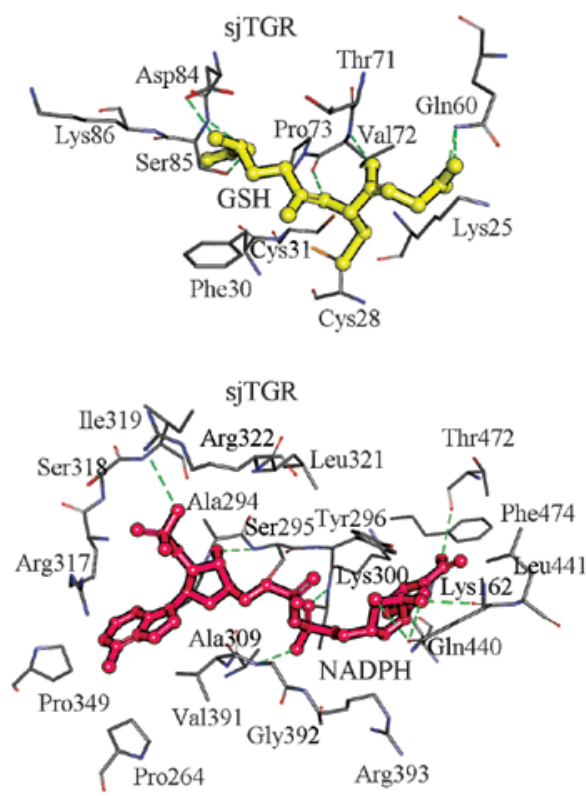

Figure 2. Binding modes of native substrates (A) GDS, (B) GSH and (C) NADPH in smTGR (left) and sjTGR (right). Residues contacting substrates (within $0.5 \mathrm{~nm}$ ) are shown as sticks. Hydogen bonds are represented by dashed green lines. GDS, oxidised glutathione; GSH, glutathione; smTGR, thioredoxin glutathione reductase from Schistosoma mansoni; sjTGR, thioredoxin glutathione reductase from Schistosoma japonicum.

cavity were $>2.3$, higher than those in the Grx domain or the NADPH-binding site. For sjTGR, the values of the docking ratio in the TR active centre were $>1.9$ (Fig. 3B). These values were generally lower than those for smTGR, indicating that the compounds were less effective sjTGR inhibitors. The data suggested that it is necessary to design effective inhibitors for the specific TGRs from different Schistosoma species.

The docking ratios in the TR active cavity of sjTGR and smTGR were both higher than those in the Grx domain or the NADPH-binding site, suggesting that these compounds may exert their inhibitory effects mainly through interactions in the TR active cavity, with smaller effects on the Grx domain and NADPH-binding site. The TR active cavity may thus be the favoured binding site for these compounds, which may effectively prevent the native substrate from accessing this binding site and disrupting the electron transfer in the TGRs. The following section focuses on the binding mode of compounds in the TR active cavity.

Binding mode of compounds in the active site of the GR/TR domain

Oxadiazole 2-oxides. Simeonov et al (12) identified 39 molecules with an $\mathrm{IC}_{50}<10 \mu \mathrm{M}$ from a quantitative high-throughput screen (qHTS) of 71,028 compounds. The $\mathrm{IC}_{50}$ values of the oxadiazole 2-oxides (compounds 1 and 2) against smTGR in the initial qHTS test were 8.7 and $9.2 \mu \mathrm{M}$, respectively, although compound 2 was much more active $\left(\mathrm{IC}_{50}=0.02 \mu \mathrm{M}\right)$ in the TGR assays than compound $1\left(\mathrm{IC}_{50}=1.8 \mu \mathrm{M}\right)(11)$. According to Sayed et al (13), however, the $\mathrm{IC}_{50}$ values of compounds 1,2 and 3 against GR activity were $0.32,0.51$ and $12.5 \mathrm{mM}$, respectively.

The present docking results showed that the oxadiazole 2-oxides occupy a similar position at the dimer interface in the two TGRs (Fig. 4A-C), making contacts with Ile160, Lys163 His204 (from one subunit) and Phe505', Lys506', Leu507', Glu508', His571'-Cys574' and Thr577' (from another subunit).

It was found that compound 1 adopted the same conformation in both smTGR and sjTGR (Fig. 4A) and had similar free binding energies (6.63 and $6.45 \mathrm{kcal} / \mathrm{mol}$, respectively). The cyano group was in close proximity to Pro572' (both $2.8 \AA$ in the two TGRs), the $\mathrm{N}$-atom of the oxadiazole formed a hydrogen bond with Leu508' (both $3.0 \AA$ ) and the phenyl ring made an arene-H interaction with Lys506' (both $4.2 \AA$ ). These results indicate that compound 1 may also inhibit sjTGR and are consistent with the conclusion of Sayed et al (13) that this compound is also active against Schistosoma japonicum. 
$\mathbf{A}$

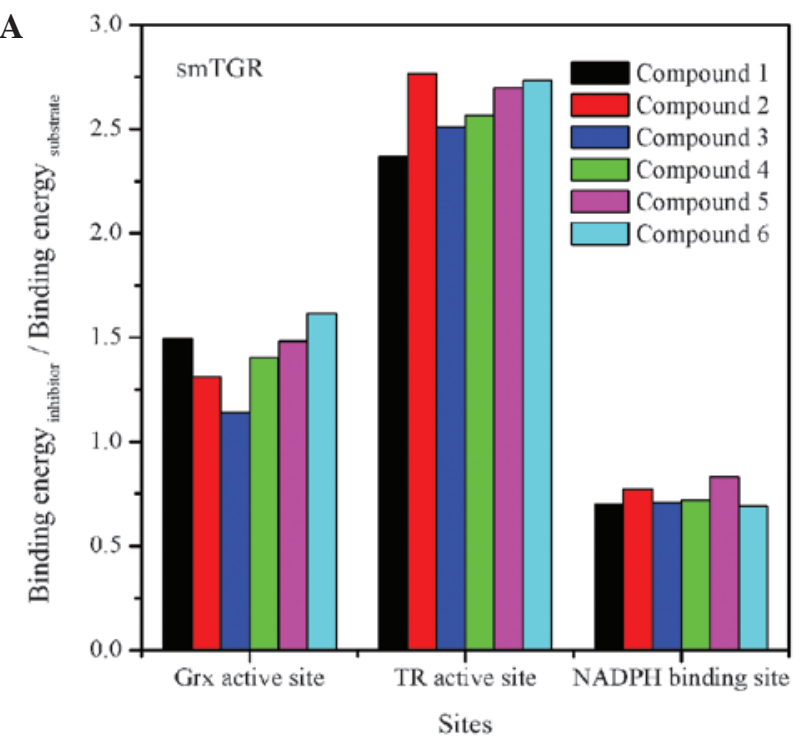

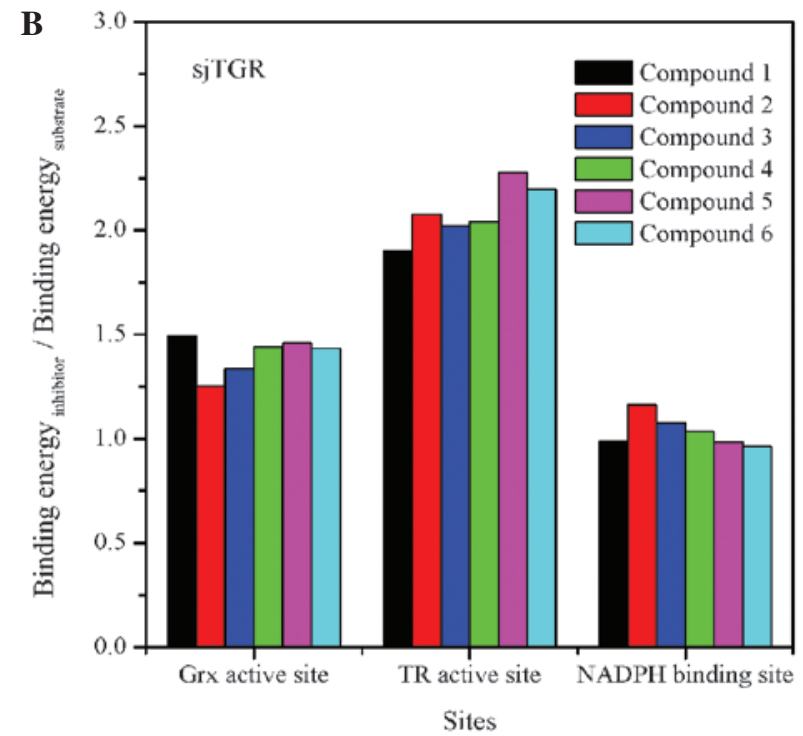

Figure 3. Ratios of the free binding energies of the inhibitors to the free binding energies of the native substrates glutathione, oxidised glutathione and NADPH in the corresponding Grx active site, TR active site and NADPH binding site, respectively. (A and B) Docking ratio of test molecules in (A) smTGR and (B) sjTGR. TR, thioredoxin reductase; Grx, glutaredoxin; smTGR, thioredoxin glutathione reductase from Schistosoma mansoni; sjTGR, thioredoxin glutathione reductase from Schistosoma japonicum.

A

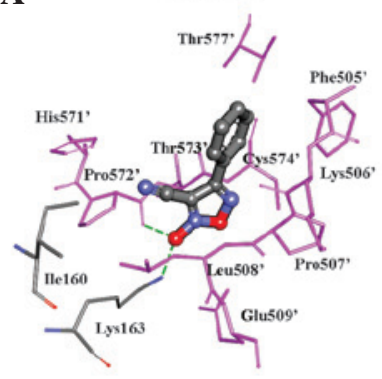

C

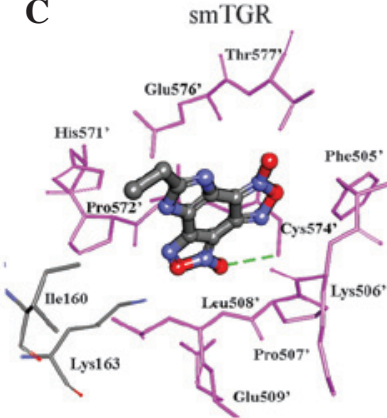

E

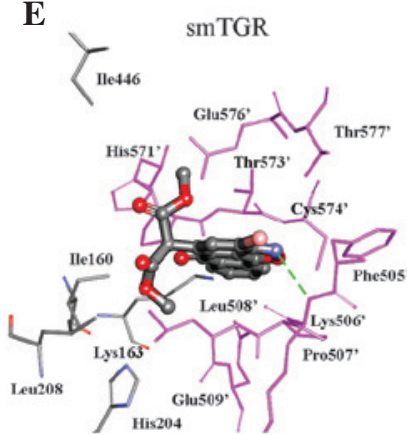

sjTGR
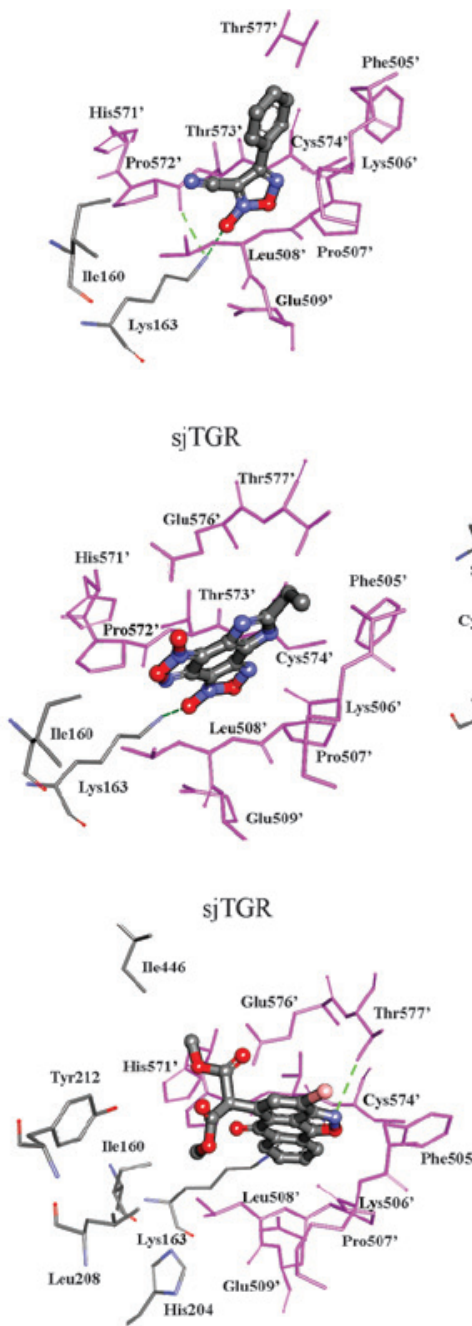

B

smTGR

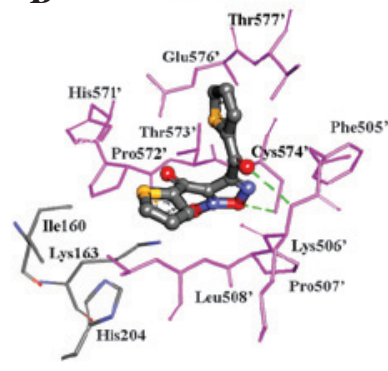

D

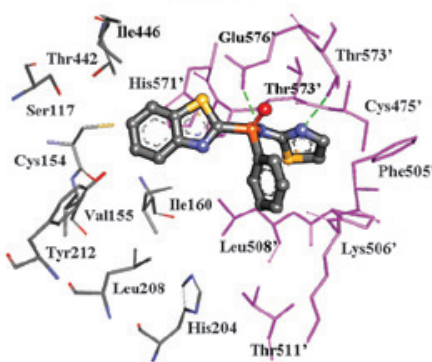

F

F $\quad$ smTGR

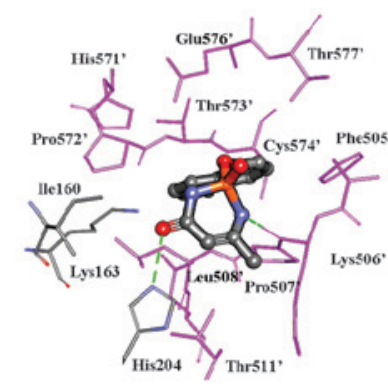

sjTGR

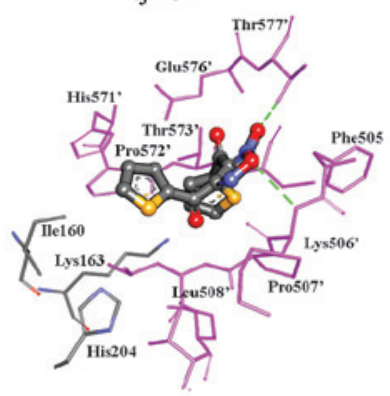

sjTGR

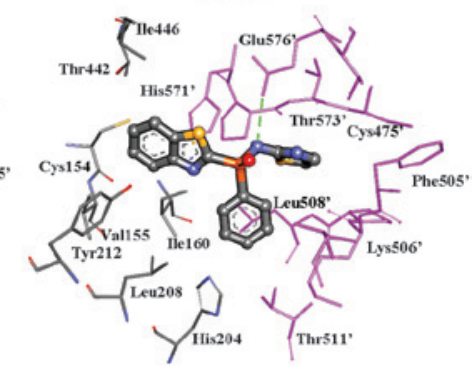

sjTGR

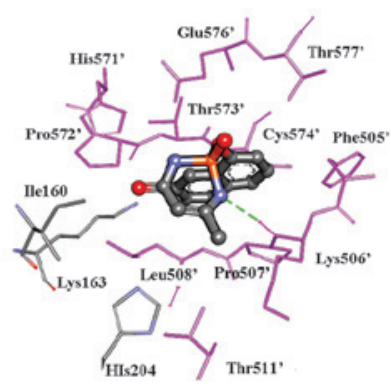

Figure 4. Binding modes of six inhibitors in the TR active cavity of smTGR and sjTGR. (A-F) Compound 1-6, respectively. Residues interacting with the inhibitors are shown as thin sticks and inhibitors are represented by sticks and balls. Hydrogen bonds are represented by dashed green lines. TR, thioredoxin reductase; smTGR, thioredoxin glutathione reductase from Schistosoma mansoni; sjTGR, thioredoxin glutathione reductase from Schistosoma japonicum. 
Compound 2 has two large acetylthiophene groups in the oxadiazole ring. In smTGR, the carbonyl O of one acetylthiophene group and the $\mathrm{O}$-atom of the oxadiazole formed hydrogen bonds with Lys506' and Cys574', respectively, stabilising its conformation in the active site. The best docking conformation of compound 2 in sjTGR, however, was different from that in smTGR. The $\mathrm{O}$-atom of the oxadiazole ring formed a hydrogen bond with Lys506' and N-O with Thr577' of sjTGR (Fig. 4B).

The compound 3 molecule exists in a rigid plane. It was found to bind in the same position at the dimer interface of the TGRs by interacting with the Leu160 and Lys163 residues and peptide fragments $505^{\prime}-508^{\prime}$ and $571^{\prime}-577^{\prime}$ in the other chains (Fig. 4C). We cannot explain why Sayed et al (13) found compound 3 to be less active against GR and TR than compound 1 , since the free binding energy of compound 3 was better than that of compound 1 in both TGRs.

Phosphinic acid amide. N-(benzothiazol-2-yl-phenyl-phosphoryl)-1,3-thiazol-2-amine (compound 4) has been identified as an inhibitor of smTGR and has also been found to be effective against the parasite $(11,12)$. Simeonov et al (12) indicated that the role of the benzothiazole group is critical for the inhibitory activity.

In the present case, the phosphinic acid amide accessed the same pocket as the oxadiazole 2-oxides (Fig. 4A-C). Both in smTGR and sjTGR, the phosphinic acid amide formed hydrogen bonds with the carboxyl group of Glu576'. The benzothiazole ring reached out to the redox couple (Cys154-Cys159) and was surrounded by Cys154, Val155, Ile160, Leu208, Thr442 and Ile446. Two peptide segments of the TGRs, 505'-508' and 572'-577', stabilised the thiazole group of compound 4 in the TGRs through van der Waals forces and hydrophobic interactions (Fig. 4D).

Isoxazolone. Compound 5, the isoxazolone dimethyl 2-[3-bromo-6-oxo-6H-anthra (1,9-cd)isoxazol-5-yl] malonate, adopted a similar conformation in both smTGR and sjTGR. The binding pocket for isoxazolone is mainly composed of residues from two subunits and involves residues 160-163, 204, 208, 212, 442, 446, 505'-509' and 571'-577' (Fig. 4E). The ligand is stabilised in the active site of the proteins, mainly through van der Waals forces and hydrophobic interactions. In the TGRs, the isoxazolone exhibited a high binding score, indicating that it may be an effective inhibitor. This finding is in agreement with the data presented by Simeonov et al (12), in which the isoxazolone exhibited a low $\mathrm{IC}_{50}$ against smTGR in the qHTS. The isoxazolone exhibited the lowest free binding energy of all the compounds with sjTGR and its binding score for docking into sjTGR was even higher than that for docking into smTGR, therefore suggesting that isoxazolone may also be active against sjTGR.

Phosphoramidite. Compound 6, the phosphoramidite 6-methyl-2-(naphthalen-1-yloxy)-2,3-dihydro-1,3,2-diazaphosphinin-4(1H)-one 2-oxide, was identified as an inhibitor of smTGR by both the qHTS and the TGR assay (12). As with the other compounds, the best binding site of the phosphoramidite, both in smTGR and in sjTGR, involved Ile160, Lys163, His204, Phe505'-Leu508' and Pro572'-Thr577'. Lys506' and His571' were within hydrogen bonding distance of the ligand (Fig. 4F). The estimated free binding energies of the phosphoramidite were $-7.66 \mathrm{kcal} / \mathrm{mol}$ with smTGR and $-7.44 \mathrm{kcal} / \mathrm{mol}$ with sjTGR (Tables II and III). These data suggest that the phosphoramidite may also be active against Schistosoma japonicum.

Conclusion. In order to explore the interaction mechanism of these compounds, six previously identified TGR inhibitors were docked into the NADPH binding site, the TR active cavity and the Grx active site of smTGR, as determined by the X-ray structure (Schistosoma mansoni), and into the structure of sjTGR (Schistosoma japonicum), as constructed by homology modelling. The present results indicate that the favourite binding site of inhibitors is the TR active cavity surrounded by an FAD-binding site, a redox-active Cys154/Cys159 pair from one subunit and a $\mathrm{C}$-terminal peptide from the other subunit. Although these inhibitors are structurally quite different, the best binding positions of all the compounds in smTGR and sjTGR were similar in the GDS binding site. Two peptide fragments from another subunit, Phe505'-Leu508' and Pro572'-Thr577', played a critical role in the interactions with the inhibitors. The data also suggest that the inhibitors are generally marginally less effective against sjTGR than against smTGR, implying that it would be necessary to design inhibitors specific for the TGRs from different Schistosoma species. An enhanced understanding of the binding mechanisms of potential inhibitors of Schistosoma TGRs would facilitate structure-based ligand design.

\section{Acknowledgements}

This study was supported by grants from the Jiangsu Provincial Nature Science Foundation (grant no. BK2011573), the Ministry of Science and Technology (grant nos. 2012AA020304 and 2012ZX09401012), the National Undergraduate Innovation Program (grant no. J1103512) and the National Natural Science Foundation of China (grant no. J1210026).

\section{References}

1. Engels D, Chitsulo L, Montresor A and Savioli L: The global epidemiological situation of schistosomiasis and new approaches to control and research. Acta Trop 82: 139-146, 2002.

2. Mbanefo EC, Huy NT, Wadagni AA, Eneanya CI, Nwaorgu O and Hirayama K: Host determinants of reinfection with schistosomes in humans: A systematic review and meta-analysis. PLoS Negl Trop Dis 8: e3164, 2014.

3. Hung YW and Remais J: Quantitative detection of Schistosoma japonicum cercariae in water by real-time PCR. PLoS Negl Trop Dis 2: e337, 2008.

4. Doenhoff MJ, Cioli D and Utzinger J: Praziquantel: Mechanisms of action, resistance and new derivatives for schistosomiasis. Curr Opin Infect Dis 21: 659-667, 2008.

5. Wang W, Dai JR, Li HJ, Shen XH and Liang YS: Is there reduced susceptibility to praziquantel in Schistosoma japonicum? Evidence from China. Parasitology 137: 1905-1912, 2010.

6. Melman SD, Steinauer ML, Cunningham C, Kubatko LS, Mwangi IN, Wynn NB, Mutuku MW, Karanja DM, Colley DG, Black CL, et al: Reduced susceptibility to praziquantel among naturally occurring Kenyan isolates of Schistosoma mansoni. PLoS Negl Trop Dis 3: e504, 2009.

7. Alger HM and Williams DL: The disulfide redox system of Schistosoma mansoni and the importance of a multifunctional enzyme, thioredoxin glutathione reductase. Mol Biochem Parasitol 121: 129-139, 2002.

8. Kuntz AN, Davioud-Charvet E, Sayed AA, Califf LL, Dessolin J, Arnér ES and Williams DL: Thioredoxin glutathione reductase from Schistosoma mansoni: An essential parasite enzyme and a key drug target. PLoS Med 4: e206, 2007. 
9. Angelucci F, Dimastrogiovanni D, Boumis G, Brunori M, Miele AE, Saccoccia F and Bellelli A: Mapping the catalytic cycle of Schistosoma mansoni thioredoxin glutathione reductase by X-ray crystallography. J Biol Chem 285: 32557-32567, 2010.

10. Williams DL, Bonilla M, Gladyshev VN and Salinas G: Thioredoxin glutathione reductase-dependent redox networks in platyhelminth parasites. Antioxid Redox Signal 19: 735-745, 2013.

11. Prast-Nielsen S, Huang HH and Williams DL: Thioredoxin glutathione reductase: Its role in redox biology and potential as a target for drugs against neglected diseases. Biochim Biophys Acta 1810: 1262-1271, 2011.

12. Simeonov A, Jadhav A, Sayed AA, Wang Y, Nelson ME, Thomas CJ, Inglese J, Williams DL and Austin CP: Quantitative high-throughput screen identifies inhibitors of the Schistosoma mansoni redox cascade. PLoS Negl Trop Dis 2: e127, 2008

13. Sayed AA, Simeonov A, Thomas CJ, Inglese J, Austin CP and Williams DL: Identification of oxadiazoles as new drug leads for the control of schistosomiasis. Nat Med 14: 407-412, 2008.

14. Morris GM, Huey R, Lindstrom W, Sanner MF, Belew RK, Goodsell DS and Olson AJ: AutoDock4 and AutoDockTools4: Automated docking with selective receptor flexibility. J Comput Chem 30: 2785-2791, 2009.

15. Krissinel E and Henrick K: Inference of macromolecular assemblies from crystalline state. J Mol Biol 372: 774-797, 2007.
16. de Beer TA, Berka K, Thornton JM and Laskowski RA: PDBsum additions. Nucleic Acids Res 42: D292-D296, 2014.

17. Laskowski RA, Hutchinson EG, Michie AD, Wallace AC, Jones ML and Thornton JM: PDBsum: A Web-based database of summaries and analyses of all PDB structures. Trends Biochem Sci 22: 488-490, 1997.

18. Stoll VS, Simpson SJ, Krauth-Siegel RL, Walsh CT and Pai EF: Glutathione reductase turned into trypanothione reductase: Structural analysis of an engineered change in substrate specificity. Biochemistry 36: 6437-6447, 1997.

19. Angelucci F, Miele AE, Boumis G, Dimastrogiovanni D, Brunori $M$ and Bellelli A: Glutathione reductase and thioredoxin reductase at the crossroad: The structure of Schistosoma mansoni thioredoxin glutathione reductase. Proteins 72: 936-945, 2008.

20. Sharma M, Khanna S, Bulusu G and Mitra A: Comparative modeling of thioredoxin glutathione reductase from Schistosoma mansoni: A multifunctional target for antischistosomal therapy. J Mol Graph Model 27: 665-675, 2009.

21. Song L, Li J, Xie S, Qian C, Wang J, Zhang W, Yin X, Hua Z and $\mathrm{Yu} \mathrm{C}$ : Thioredoxin glutathione reductase as a novel drug target: Evidence from Schistosoma japonicum. PLoS One 7: e31456, 2012.

22. Rai G, Thomas CJ, Leister W and Maloney DJ: Synthesis of oxadiazole-2-oxide analogues as potential antischistosomal agents. Tetrahedron Lett 50: 1710-1713, 2009. 